\title{
Vigilancia activa en carcinoma papilar tiroideo de bajo riesgo (parte I): concepto, epidemiología y situación actual en el mundo
}

\section{Active surveillance of low-risk papillary thyroid carcinoma (part I): concept, epidemiology and current situation in the world}

\author{
Mario Tapia C. ${ }^{1,4}$, Felipe Cardemil M..$^{2,3}$
}

${ }^{1}$ Servicio de Otorrinolaringología, Complejo Asistencial Dr Víctor Ríos Ruiz. Los Ángeles, Chile.

${ }^{2}$ Departamento de Oncología Básico-Clínica y Departamento de Otorrinolaringología, Facultad de Medicina, Universidad de Chile. Santiago, Chile

${ }^{3}$ Departamento de Otorrinolaringología, Clínica Las Condes. Santiago, Chile.

${ }^{4}$ Head and Neck Surgical Oncology Department, Peter MacCallum Cancer Centre. Melbourne, Australia

Los autores declaran no tener conflictos de interés.

Recibido el 6 de agosto de

2020. Aceptado el 7 de octubre de 2020 .

Correspondencia: Mario Tapia C

Servicio de

Otorrinolaringología

Complejo Asistencial Dr. Víctor Ríos Ruiz.

Los Ángeles, Chile Email: mtapiac21@gmail.com

\section{Resumen}

En las últimas décadas, la creciente identificación de nódulos tiroideos ha dado lugar a un incremento dramático en el diagnóstico y tasa de incidencia del cáncer de tiroides, sin embargo, su mortalidad se ha mantenido estable. La mayor fracción de este incremento es atribuible al creciente uso de tecnologías de diagnóstico, que ha identificado un número cada vez mayor de cánceres papilares de tiroides (CPT), en su gran mayoría pequeños y de bajo riesgo clínico. La mayoría de estos tumores no generarán morbilidad o mortalidad a pesar de no ser tratados. La práctica clínica ha evolucionado hacia un tratamiento menos agresivo de los CPTs. La vigilancia activa (VA) ha surgido como una nueva alternativa terapéutica dentro del algoritmo de tratamiento, que tiene como objetivo reconocer a un potencial grupo minoritario de pacientes en quienes sus carcinomas papilares tiroideos sí progresarán clínicamente y, por lo tanto, en ellos un tratamiento quirúrgico resulte en mayor beneficio que perjuicio. En esta revisión nos planteamos como objetivo describir la epidemiología del carcinoma papilar tiroideo, el concepto de vigilancia activa en esta área y los resultados clínicos obtenidos mediante este abordaje terapéutico en las distintas experiencias realizadas en el mundo hasta la fecha.

Palabras clave: Vigilancia activa, carcinoma papilar tiroideo, bajo riesgo, microcarcinoma papilar tiroideo, cáncer de tiroides.

\begin{abstract}
In recent decades, the increasing identification of thyroid nodules has led to a dramatic increase in the diagnosis and incidence rate of thyroid cancer. However, their mortality has remained stable. The largest fraction of this increase is attributable to the growing use of diagnostic technologies, which has identified an increasing number of mostly small, low clinical risk papillary thyroid cancers (PTC). Most of these tumors will not generate morbidity or mortality despite being untreated. Clinical practice has evolved towards a less aggressive treatment of PTC's. Active surveillance (AS) has emerged as a new therapeutic alternative within the treatment algorithm, which aims to recognize a potential minority group of patients in whom their thyroid papillary carcinoma will progress clinically, and therefore in whom surgical treatment will result in greater benefit than harm. In this review we aim to describe the epidemiology of papillary thyroid carcinoma, the concept of active surveillance in this area and the clinical results obtained by this therapeutic approach in the different experiences carried out in the world until now.
\end{abstract}

Keywords: Active surveillance, papillary thyroid carcinoma, low risk, papillary thyroid microcarcinoma, thyroid cancer. 


\section{Introducción}

El cáncer de tiroides es el tumor maligno más común del sistema endocrino, entre los cuales, los carcinomas papilares representan más del $80 \%$ de ellos ${ }^{1}$. Este grupo representa el tipo histológico menos agresivo de cáncer en esta glándula, caracterizados por una tasa de crecimiento lento y un curso indolente, con una supervivencia general de aproximadamente $97 \%$ a 10 años en pacientes tratados con tiroidectomía y terapias adyuvantes ${ }^{2}$.

El microcarcinoma papilar de tiroides (MCPT) en su definición original corresponde a un CPT que mide igual o menos de $10 \mathrm{~mm}$ de diámetro máximo, independientemente de que estuviesen presentes características de alto riesgo como extensión extratiroidea, o la presencia de metástasis en ganglios linfáticos y a distancia ${ }^{3}$. Los MCPT con características de alto riesgo pueden requerir un tratamiento extenso que involucre una tiroidectomía total (TT) asociado a disección profiláctica/terapéutica de los ganglios linfáticos cervicales, terapia adyuvante/terapéutica con yodo radiactivo (RAI), o supresión de la hormona estimulante de la tiroides (TSH) bajo una cuidadosa vigilancia de los niveles de tiroglobulina y de anticuerpos de tiroglobulina. En la actualidad, el principal problema clínico es determinar el manejo de los MCPT que no presentan estas características de alto riesgo, con objeto de administrar un tratamiento proporcional y evitar terapias innecesarias con sus consiguientes efectos adversos, más aún considerando que estos carcinomas son los principales responsables del reciente aumento de la incidencia de cáncer de tiroides a nivel mundial ${ }^{3}$.

Durante décadas el tratamiento estándar de los CPT ha sido la resolución quirúrgica, sin embargo, la vigilancia activa (VA) ha surgido como una nueva alternativa terapéutica dentro del algoritmo de tratamiento de estos tumores, que tiene como objetivo reconocer a un potencial grupo minoritario de pacientes, en quienes sus carcinomas papilares tiroideos progresarán clínicamente $\mathrm{y}$, por lo tanto, en ellos un tratamiento quirúrgico sí resultaría en un mayor beneficio que perjuicio ${ }^{2,3}$. Además, la realización de una cirugía diferida en los pacientes adecuadamente seleccionados con $\mathrm{CPT}$, no se ha asociado con un mayor riesgo de morbilidad o recurrencia en comparación con la intervención temprana o inmediata ${ }^{2,3}$. Las experiencias acumuladas con este abordaje terapéutico inicialmente en Asia, y recientemente en distintos continentes, han demostrado que la VA es una alternativa segura para el manejo de CPT de bajo riesgo. En esta revisión se describirá la epidemiología del carcinoma papilar tiroideo, el concepto de vigilancia activa en esta área y los resultados clínicos obtenidos mediante este abordaje terapéutico en las distintas experiencias realizadas en el mundo hasta la fecha.

\section{Epidemiología}

La incidencia del cáncer de tiroides ha aumentado en gran parte del mundo, especialmente en naciones desarrolladas ${ }^{4}$. Vaccarella y cols. reportaron el aumento de la incidencia de cáncer de tiroides en países de altos ingresos y notaron aumentos dramáticos en el diagnóstico en Estados Unidos, Italia, Francia y otras naciones desarrolladas ${ }^{4}$. El aumento más dramático se ha observado en Corea del Sur donde la incidencia entre las mujeres aumentó de 30 a 120 casos por cada 100.000 mujeres entre los años 2003 y $2007^{4}$.

La creciente incidencia del cáncer de tiroides en todo el mundo se ha vinculado al uso más amplio de técnicas de diagnóstico como la ecografía o ultrasonido de cuello asociado con aspiración con aguja fina (PAAF) de los pequeños nódulos encontrados incidentalmente ${ }^{5-10}$. En Estados Unidos los nuevos diagnósticos de cáncer de tiroides se han triplicado en las últimas cinco décadas ${ }^{11}$. Si bien entre las hipótesis que explican estas tendencias en aumento se han incluido las etiologías ambientales y genéticas, un conjunto cada vez más amplio de evidencia ha confirmado que la principal contribución a esta incidencia en aumento ha sido el desarrollo y el uso más amplio de las modalidades diagnósticas mencionadas ${ }^{6,12}$. Se ha estimado que el $70 \%$ a $80 \%$ de los casos en los Estados Unidos, y el 50\% a 90\% de los casos en otras naciones desarrolladas no habrían llegado a causar síntomas, daño o muerte si nunca se hubiesen diagnosticado, un fenómeno que se denomina sobrediagnóstico ${ }^{4}$. Este aumento de la incidencia se atribuye casi en su 
totalidad al CPT y principalmente de tumores de menos de $2 \mathrm{~cm}$ de tamaño $0^{5,7,11,12}$.

A pesar del considerable aumento de la incidencia, la tasa de mortalidad atribuible al cáncer de tiroides ha permanecido sin cambios significativos ${ }^{11}$. En Estados Unidos la incidencia del CPT aumentó 2,4 veces entre 1973 y 2002 y 2,9 veces entre 1975 y 2009 , con tasas de mortalidad estables ${ }^{11,13}$. Los análisis de los datos de registros nacionales de cáncer de Estados Unidos han revelado un discreto aumento de la mortalidad por cáncer de tiroides en un subconjunto de pacientes, de 0,06 por 100.000 en dos décadas, en el contexto de un aumento paralelo de la incidencia que asciende a 8,2 por $100.000^{1}$, lo que sugiere que casi todos los casos adicionales que se están diagnosticando en la actualidad no resultan ser en última instancia letales ${ }^{11}$. En Corea del Sur, la incidencia del carcinoma de tiroides aumentó hasta 15 veces entre 1993 y 2001 como resultado del programa nacional de detección del cáncer de tiroides mediante ultrasonido y PAAF; sin embargo, la tasa de mortalidad del carcinoma de tiroides no varió $^{14}$. También se han notificado fenómenos epidemiológicos similares en Italia, el Reino Unido, Australia y los países nórdicos ${ }^{1}$.

\section{Patología}

El cáncer de tiroides frecuentemente es asintomático, y su hallazgo ha sido comúnmente identificado en grandes estudios en los cuales se realizaron autopsias en pacientes que no presentaban enfermedad tiroidea conocida ${ }^{15,16}$. Se ha reportado una prevalencia de cánceres ocultos de tiroides de hasta un $30 \%$ en un estudio muy citado de Finlandia ${ }^{15}$, y en otros, tasas de CPT subclínico entre 5\% a $30 \%{ }^{16}$. Otra metodología utilizada para estimar la prevalencia del carcinoma papilar oculto en pacientes más jóvenes ha sido examinar las glándulas tiroides extraídas por enfermedades benignas. En estos estudios, se ha reportado una prevalencia de microcarcinomas pequeños de hasta el 10,5\%, sin evidencia de enfermedad clínica en este grupo de pacientes en seguimientos de más de 5 años ${ }^{17}$.

Con el desarrollo del ultrasonido y la aspiración con aguja fina guiada por ultrasonido, los MCPT se han detectado y diagnosticado durante tamizaje masivo y estudios imagenológicos para otras enfermedades, como el ultrasonido de la arteria carótida para evaluar esclerosis arterial y tomografía computarizada de tórax para evaluar patologías pulmonares o metástasis pulmonar de otros carcinomas. En un estudio realizado por Takebe y cols. ${ }^{18}$, en el cual se realizó ultrasonido a mujeres sanas mayores a 30 años, se detectó y diagnosticó carcinoma de tiroides en el 3,5\% de las mujeres, de las cuales el 85\% medían menos de $15 \mathrm{~mm}$. Esta incidencia fue similar a la de los estudios de autopsia y 1.000 veces mayor que la prevalencia de carcinoma tiroideo clínico en las mujeres japonesas notificadas en ese momento $(3,1 \text { por cada } 100.000 \text { habitantes })^{18}$. Estas conclusiones sugieren que la mayoría de los MCPT son asintomáticos, no crecen o crecen muy lentamente, y que muchas personas viven con ellos sin saber que son portadores, a menos que se sometan a estudios de diagnóstico por imágenes o PAAF. Esto ha generado dudas sobre si la detección, el diagnóstico y el tratamiento de estos tumores resulta beneficioso para los pacientes. En conjunto, estos datos indican que la verdadera incidencia del carcinoma tiroideo subclínico es mucho mayor que la prevalencia de la enfermedad clínica ${ }^{15-18}$.

Como se mencionó anteriormente, los carcinomas ocultos tienden a ser predominantemente CPT pequeños ${ }^{13,14} y$ más específicamente MCPT. Los MCPT tienden a ser indolentes con bajas tasas de metástasis locorregionales y a distancia, y tienen una excelente supervivencia específica de la enfermedad de casi el 100\% a 10 años $^{19-21}$. Al analizar estos datos en conjunto con la comprensión de que cerca de ninguno de los casos representados por la creciente incidencia de CPT parecen resultar en un aumento significativo de la tasa de mortalidad, estas tendencias identificadas pueden atribuirse razonablemente a una mayor sensibilidad en el diagnóstico de la enfermedad oculta y que la mayoría de estos MCPT resultan ser de bajo riesgo ${ }^{11,13}$. Así pues, es evidente que los carcinomas tiroideos relativamente pequeños están presentes regularmente en adultos sanos, y muchos de ellos nunca tendrán repercusiones negativas en su bienestar y calidad de vida ${ }^{15-21}$.

Actualmente no existen ensayos clínicos controlados aleatorizados que comparen el tratamiento inicial agresivo en CPT con tra- 
tamientos menos agresivos. Por lo tanto, las decisiones relativas a la intervención suelen guiarse por datos de estudios retrospectivos o estudios poblacionales. En el pasado se defendió enérgicamente el tratamiento agresivo de los CPT en un intento por mejorar los resultados de supervivencia de los pacientes con esta patología. Sin embargo, estudios posteriores no han evidenciado ninguna ventaja estadísticamente significativa de supervivencia para la tiroidectomía total al compararlo con hemitiroidectomía entre los pacientes con tumores de menos de $4 \mathrm{~cm}$ de tamaño ${ }^{22-27}$. Sobre la base de esta nueva evidencia, el año 2016 la guía de la Asociación Americana de Tiroides (ATA) se actualizó para permitir ofrecer un tratamiento más conservador en pacientes cuidadosamente seleccionados ${ }^{28,29}$. Recomendaciones anteriores de tiroidectomía total con ablación de yodo radiactivo (RAI) posoperatoria para todos los casos de cáncer de tiroides han sido reemplazados por un nuevo enfoque estratificado de riesgo, que utiliza una cirugía menos agresiva (lobectomía o hemitiroidectomía) sin requerir el uso rutinario de RAI posoperatoria en los CPT de bajo y mediano riesgo ${ }^{28,29}$.

\section{Vigilancia activa}

En las últimas dos décadas desde la publicación de los artículos japoneses y más aún desde la actualización de la guía ATA el año 2016, se ha sugerido la VA como una alternativa terapéutica para los MCPT de bajo riesgo en lugar de la cirugía inmediata ${ }^{30-32}$. Por definición la VA significa aplicar por largo plazo modalidades de diagnóstico periódicas y regulares para evaluar los cambios en el estado de la enfermedad sin tratamiento hasta que la progresión de la enfermedad resulte clínicamente evidente ${ }^{33,34}$. La VA ha sido estudiada más ampliamente en el cáncer de próstata ${ }^{35}$. Los cánceres de próstata son biológicamente indolentes en muchos casos, y las complicaciones quirúrgicas como la incontinencia urinaria y la disfunción eréctil son relativamente comunes. Además, el cáncer de próstata suele desarrollarse en pacientes de edad avanzada ${ }^{33,36}$. En vista de que la mayoría de los MCPT también tienen una naturaleza muy indolente, la cirugía inmediata podría no estar absolutamente indicada, a pesar de que las tasas reportadas de complicaciones quirúrgicas sean bajas en cirujanos experimentados $\mathrm{y}$ hospitales con alto volumen ${ }^{31}$.

La vigilancia activa en MCPT fue por primera vez propuesta por Akira Miyauchi en 1993, basándose en los resultados de CPT ocultos encontrados en los estudios de autopsia y estudios de tamizaje mediante ultrasonido ${ }^{16,18}$. La VA de los MCPT de bajo riesgo se inició como un estudio de cohorte prospectivo en 1993 en el Hospital de Kuma en Japón, bajo las hipótesis de que la mayoría de los MCPT se mantienen en un tamaño pequeño, que la VA era la única estrategia para evaluar si los MCPT presentaban crecimiento, y además, que en el caso de observarse progresión de la enfermedad como crecimiento tumoral o desarrollo de metástasis ganglionares durante la VA la cirugía diferida no generaría mayor morbimortalidad y que, por lo tanto, la cirugía inmediata o temprana resultaría más perjudicial que beneficiosa para la gran mayoría de estos pacientes ${ }^{31}$. Su colega Yashuhiro Ito continuó esta práctica en el mismo hospital, al igual que Iwao Sugitani en 1995 en el Hospital del Instituto del Cáncer de Tokio bajo las mismas hipótesis. Estos dos hospitales en conjunto tienen la mayor y más larga experiencia en ofrecer VA a pacientes con MCPT ${ }^{29}$. En vista de sus resultados exitosos, la estrategia de VA para MCPT fue aceptada como abordaje terapéutico en las guías de la Asociación Japonesa de Cirujanos Endocrinos y la Sociedad Japonesa de Cirugía Tiroidea (actualmente Asociación Japonesa de Cirugía Endocrina [JAES] ${ }^{37}$ y luego, fuertemente recomendada en la guía revisada de la JAES en $2018^{38}$. La guía ATA 2015 también estableció que es posible considerar Vigilancia Activa en $\mathrm{MCPT}^{29} \mathrm{y}$, desde entonces, se han comenzado a reportar resultados de VA de otros países.

Hasta la fecha, no ha habido ensayos aleatorios controlados prospectivos que evalúen la VA en comparación con la cirugía inicial para pacientes con CPT. Sin embargo, como se ha indicado anteriormente, la VA se ha utilizado en grandes series no aleatorizadas de pacientes, prospectivas y retrospectivas, con datos sóli$\operatorname{dos}^{30,39-42}$. El primer estudio sobre la vigilancia activa en el Hospital de Kuma evidenció que más del 70\% de los MCPT no aumentaban su tamaño ni desarrollaban adenopatías 
cervicales $^{31}$. En su segundo informe en 2010, emplearon un análisis de secuencia temporal e identificaron que las tasas de crecimiento tumoral a 5 y 10 años correspondían a 6,4\% y $15,9 \%$ respectivamente, y que las tasas de metástasis ganglionar regional a 5 y 10 años fueron de $1,5 \%$ y $3,4 \%$, respectivamente ${ }^{32}$. En 2014 , los resultados de la mayor serie prospectiva de pacientes sometidos a VA en un período de 22 años en el Hospital Kuma fueron publicados por Ito y Miyauchi ${ }^{30,39}$. En este estudio de 2.153 pacientes, 1.235 de ellos se sometieron a VA en lugar de cirugía inmediata, presentando resultados oncológicos similares al grupo que recibió cirugía. Se reportó que las tasas de crecimiento tumoral acumulativas a 5 y 10 años eran del $4,9 \%$ y $8,0 \%$ y las tasas acumulativas a 5 y 10 años de metástasis ganglionar linfática de $1,7 \%$ y $3,8 \%$, respectivamente. También se realizó un análisis de subconjunto de la edad de los pacientes en el momento del diagnósti$\mathrm{Co}^{30}$. El crecimiento del tumor igual o mayor a $3 \mathrm{~mm}$ disminuyó significativamente con la edad avanzada: las tasas a 5 y 10 años fueron $9,1 \%$ y $12,1 \%$ para los pacientes de menores a 40 años, 5,0\% y 9,1\% para los pacientes entre 40 y 59 años, y $4,0 \%$ y $4,1 \%$ para los pacientes de 60 años o más. De manera similar, las tasas de metástasis ganglionar linfática estaban inversamente relacionadas con la edad de los pacientes; las tasas a 5 y 10 años fueron de 5,2\% y $16,1 \%$ para los pacientes menores a 40 años, $1,4 \%$ y $2,3 \%$ para los pacientes entre 40 a 59 años, y $0,5 \%$ para los pacientes mayor o igual a 60 años. En un análisis multivariado, sólo la edad joven (pacientes menores de 40 años) fue un predictor independiente para la progresión de CPT, mientras que la presencia de CPT múltiples y una historia familiar de carcinoma de tiroides diferenciado no lo fueron, sugiriéndose que la VA no resultaba inapropiada en pacientes con MCPT múltiple y/o historial familiar de carcinoma de tiroides diferencia$\mathrm{do}^{30}$. Además, todos los pacientes tratados mediante VA inicialmente que requirieron cirugía debido a progresión de la enfermedad fueron tratados con éxito sin complicaciones o peor resultado clínico y no se reportaron muertes en ninguno de los grupos ${ }^{30}$.

Un segundo estudio prospectivo realizado de manera similar en 230 pacientes con MCPT asintomático durante un período de hasta 17 años por Sugitani y cols. del Hospital del Instituto del Cáncer de Tokio ${ }^{40}$, identificó que la mayoría de los pacientes no presentó un cambio apreciable en el tamaño de sus tumores con tasas de crecimiento tumoral y metástasis ganglionar linfática de $7 \%$ y $1 \%$, respectivamente $^{40}$. Más importante aún, ninguno de los pacientes desarrolló extensión extratiroidea (EET) o metástasis a distancia. Doce pacientes finalmente requirieron cirugía y no se observó recurrencia en ninguno de ellos durante el seguimiento posoperatorio. Posteriormente, un grupo coreano publicó su experiencia con VA en 192 pacientes con $\mathrm{MCPT}^{41}$, reportando tasas relativamente bajas de crecimiento tumoral con 24 pacientes sometidos a cirugía tiroidea tardía, y ningún paciente presentó recurrencia durante el seguimiento.

Los resultados de estas tres series en Asia evidenciaron la seguridad de la VA como una alternativa terapéutica para este diagnóstico. También, los pacientes que necesitaron una intervención quirúrgica después de iniciar la VA, fueron sometidos a un procedimiento quirúrgico definitivo y evitaron potenciales múltiples cirugías debido a eventuales recurrencias tras la primera intervención ${ }^{39}$. Además de demostrar la seguridad de la VA, estas experiencias en Japón y Corea ayudaron a la comprensión actual de la progresión natural de los pequeños CPT de bajo riesgo. Las observaciones adicionales permitieron estratificar el riesgo de las lesiones en función de la ubicación, la relación con la cápsula del tumor y la histología.

\section{Carcinoma papilar tiroideo de bajo riesgo}

Una de las principales consideraciones en que se basa la VA es la de establecer qué pacientes tienen tumores que no progresarán. Los MCPT con características de alto riesgo como metástasis clínica en los ganglios linfáticos y/o metástasis a distancia son candidatos para el tratamiento quirúrgico y terapia con RAI adyuvante/terapéutica, asociado a otros posibles tratamientos ${ }^{29}$. Los MCPT sintomáticos con parálisis de cuerdas vocales debido a la invasión del nervio laríngeo recurrente, o los que presenten signos de invasión de la tráquea en los estudios por imágenes también deben ser 
tratados de manera quirúrgica. Además, los MCPT sospechosos citológicamente de malignidad de alto grado, como las variantes de células altas también son candidatos fuertes para tratamiento quirúrgico temprano ${ }^{29}$. Incluso si no se detecta ninguna de las características de alto riesgo mencionadas, los MCPT que posiblemente invadan la tráquea y/o estén situados a lo largo del nervio laríngeo recurrente no son adecuados para VA. Por lo tanto, es importante evaluar cuidadosamente la relación entre la ubicación del tumor y la invasión de la tráquea y/o el nervio laríngeo recurrente.

Para la evaluación de la invasión traqueal resultan útiles la medición de los ángulos formados por el cartílago traqueal y la superficie del tumor mediante ecografía de cuello y/o tomografía computada (TC) de cuello ${ }^{42}$. Entre los MCPT que tengan una dimensión igual o mayor a $7 \mathrm{~mm}$ con ángulos obtusos, el $24 \%$ mostró una invasión traqueal significativa que requirió al menos una resección laminar. $\mathrm{Al}$ evaluar la invasión del nervio laríngeo recurrente, es importante determinar que exista un borde conservado entre la superficie del tumor en relación al trayecto del nervio laríngeo recurrente $^{14}$. En un 9\% de los MCPT mayores o igual a $7 \mathrm{~mm}$ sin un borde conservado se evidenció una invasión del NLR que requirió resección de este ${ }^{42}$.

La evaluación histológica de CPT, confirmados en un estudio que involucró a 1.143 pacientes portadores de CPT de bajo riesgo tratados mediante resolución quirúrgica, reveló patrones de crecimiento expansivo e invasivo $^{42}$. Las lesiones con crecimiento expansivo mostraron márgenes agudos entre el tumor y el parénquima de la glándula circundante con una cápsula intacta, mientras que las lesiones con un patrón de crecimiento invasivo mostraron márgenes irregulares, falta de cápsula tumoral e infiltración en el parénquima de la tiroides, y tenían un mayor riesgo de recurrencia $^{42}$. Además, tener menos de 40 años resultó ser un factor predictivo independiente del crecimiento de los nódulos a lo largo del tiempo, mientras que los pacientes mayores presentaron un crecimiento infrecuente ${ }^{30,44}$.

La guía ATA de 2015 define a los siguientes pacientes con MCTP como potencialmente elegibles para VA; pacientes con MCPT de muy bajo riesgo; pacientes en los que las co- morbilidades hacen que la cirugía sea de alto riesgo; pacientes que se espera que tengan una esperanza de vida relativamente corta; o pacientes con problemas médicos o quirúrgicos que deben ser tratados antes de la cirugía de tiroides. Los pacientes deben estar dispuestos a aceptar el abordaje terapéutico mediante VA, comprender que la intervención quirúrgica puede ser necesaria en el futuro y cumplir con el programa de vigilancia periódica. Además, las características citológicas y radiológicas de los tumores, y las características clínicas de los pacientes, deben considerarse como criterios de elegibilidad para VA. Adicionalmente, se debe contar con un equipo médico especializado para vigilar a los pacientes durante la $\mathrm{VA}^{29}$.

Basándose en la actualización de la guía del 2015, Brito y cols. propusieron un marco para la estratificación de riesgo de los pacientes con CPT que pudiesen considerarse para $\mathrm{VA}^{45}$. Estos criterios clasifican a los pacientes en las categorías: "ideal”, "apropiado" e "inapropiado" para VA. Los pacientes categorizados en el primer grupo corresponden a pacientes mayores con un único tumor probable o comprobado dentro del parénquima tiroideo, lejos de la cápsula tiroidea y con márgenes bien definidos. Los pacientes categorizados como "apropiados" incluyen a pacientes más jóvenes, con enfermedad multifocal en lugares no críticos cerca de la cápsula tiroidea, o que puedan tener condiciones concurrentes que hacen más difícil el seguimiento ecográfico. Finalmente, los candidatos categorizados como "inapropiados" corresponden a pacientes con tumores en ubicaciones críticas como el nervio laríngeo recurrente, esófago o tráquea, características citológicas agresivas, evidencia radiográfica de EET, o evidencia de crecimiento y progresión de la enfermedad en estudios imagenológicos seriados.

Ito y cols. ${ }^{46}$ reportaron que la invasión de la tráquea y/o la afectación recurrente del nervio laríngeo pueden evaluarse con mayor precisión mediante la tomografía computarizada. Así, en el caso de un tumor adyacente a la tráquea o al surco traqueal, se debe aplicar una TC adicional para excluir a los candidatos inapropiados para VA.

Algunos estudios han sugerido que los CPT con un tamaño de 1 a $2 \mathrm{~cm}$ también pueden ser manejados con seguridad mediante VA. Un es- 
tudio realizado en el Memorial Sloan Kettering Cancer Center (MSKCC) incluyó a pacientes con CPT menores o iguales a $1,5 \mathrm{~cm}$ de diámetro máximo y confirmó la baja probabilidad de crecimiento de estos tumores ${ }^{47}$. Un estudio reciente de Japón comparó los resultados clínicos de VA en pacientes con enfermedad Tla y $\mathrm{T} 1 \mathrm{~b}$. Los pacientes con diagnóstico de CPT T1b con un tamaño medio de $1,2 \mathrm{~cm}$ mostraron los mismos excelentes resultados que los pacientes con CPT T1a, y sólo el 7\% mostró un aumento de diámetro mayor a $3 \mathrm{~mm}$ durante 7 años de seguimiento ${ }^{48}$. Estos estudios sugieren que los criterios de elegibilidad para la VA pueden ser ampliados.

Con respecto a las características de los pacientes, la edad parece ser el factor más importante para predecir la progresión de la MCPT durante la VA. Ito y cols. ${ }^{30}$ informaron de que se detectó un aumento de tamaño de al menos $3 \mathrm{~mm}$, el desarrollo de nuevas metástasis del LN cervical y un aumento de tamaño tumoral mayor a $12 \mathrm{~mm}$ en $4 \%, 0,5 \%$ y 2,5\% de los pacientes mayores de 60 años con MCPT, respectivamente. En contraste, en los pacientes menores a 40 años con MCPT, la incidencia fue de $12,1 \%, 16,1 \%$ y $22,5 \%$, respectivamente. Estos hallazgos sugieren que la cirugía inmediata puede ser más beneficiosa para los pacientes más jóvenes, ya que es más probable que presenten progresión de la enfermedad durante la VA. Un estudio reciente del mismo grupo reportó tasas de progresión de la enfermedad específicas de la edad en el MCPT, con tasas de progresión a 10 años de $37 \%$ para los pacientes de 20 años y del 3,5\% para los pacientes de 70 años ${ }^{49}$.

La posible invasión de la cápsula anterior o lateral de la tiroides por parte de los MCPT no es una contraindicación para la vigilancia activa $^{30}$. Incluso en el caso de requerir cirugía, la resección de los músculos anteriores infrahioideos no afecta la calidad de vida de los pacientes $^{30,39}$. La presencia de MCPT múltiples y un historial familiar de carcinoma de tiroides diferenciado tampoco son contraindicaciones para la vigilancia activa ${ }^{30,39}$. A pesar de que estos factores pueden ser factores pronósticos moderados para el CPT, la tiroidectomía total que se requiere en estos pacientes puede dar lugar a una mayor incidencia de eventos adversos significativos de la cirugía, tales como hipopa- ratiroidismo permanente y lesión de los nervios laríngeos recurrentes. Ninguno de estos factores resultó ser predictor de progresión de enfermedad según un análisis multivariado ${ }^{30}$.

Actualmente, no hay datos que indiquen que algún hallazgo molecular, como la mutación BRAF, afecte a la idoneidad de un CPT pequeño para $\mathrm{VA}^{39,47}$. Las extensas experiencias japonesas, coreanas y norteamericanas más recientes no han utilizado ningún marcador molecular como criterio de inclusión o exclusión, y se estima que una parte sustancial de los pacientes con CPT sometidos a VA en cada una de estas series habrían albergado mutaciones de BRAF. Un reciente análisis mutacional realizado por Tuttle y cols. reveló que no hay claras diferencias mutacionales entre los microcarcinomas papilares indolentes y los que desarrollan metástasis ganglionares ${ }^{47}$. Sin embargo, a medida que las experiencias aumenten y que se disponga de más casos para el análisis molecular, es posible que en el futuro se identifiquen mejores discriminadores moleculares del comportamiento clínico.

\section{Vigilancia activa fuera de Japón}

Como se señaló anteriormente, el primer estudio relacionado con la vigilancia activa de los MCPT fuera de Japón se realizó en Corea del Sur. No obstante, en este estudio se realizó una vigilancia pasiva debido a que reclutó 192 pacientes que no se sometieron a una cirugía inmediata durante más de un año, tras haber rechazado la cirugía por distintas razones o por ser portadores de otros tumores malignos no resueltos, o por presentar un riesgo elevado de recibir anestesia general. El estudio demostró que solo un 2,1\% de los MCPT presento un crecimiento basado en el tamaño máximo del tumor y un $14,1 \%$ basado en el volumen del tumor $^{41}$. En 2018, un estudio multicéntrico realizado en Corea del Sur ${ }^{50}$ de 370 pacientes con un período de seguimiento medio de 32,5 meses, reportó que $23,2 \%$ presentó un aumento en el volumen del tumor de un 50\% o más, mientras que sólo un 3,5\% presentó un aumento en el diámetro máximo de $3 \mathrm{~mm}$ o más. La incidencia de cirugía de conversión fue de $15,7 \%$, de los cuales un $37,9 \%$ fue debido a ansiedad del paciente, $32,8 \%$ debido al aumen- 
to del tamaño del tumor y $8,6 \%$ por desarrollo de metástasis ganglionares cervicales.

En 2017, Tuttle y cols. ${ }^{47}$ llevaron a cabo el primer estudio prospectivo de VA en Estados Unidos en pacientes con CPT con un diámetro máximo de $1,5 \mathrm{~cm}$ y de bajo riesgo de acuerdo con la clasificación de Brito y cols. ${ }^{45}$, en el cual 291 pacientes se sometieron a VA como alternativa a la cirugía para CPT o CPT probable (categorías V o VI de Bethesda) en base a los resultados de la ecografía y PAAF. Evaluaron los cambios tanto en el diámetro máximo como en el volumen del tumor y encontraron que, aunque sólo el 3,8\% de los tumores aumentaron de tamaño en $3 \mathrm{~mm}$ o más en su diámetro máximo, el 12,7\% de los tumores aumentaron de volumen en más de un $50 \%$. Mientras tanto, se observó una disminución del volumen mayor a $50 \%$ y un volumen estable en el $6,7 \%$ y $80,2 \%$ de los tumores, respectivamente. Los autores concluyeron que la medición del volumen tumoral resultaba ser un mejor predictor de crecimiento tumoral en comparación con el tamaño del mismo, y en consecuencia pudiese permitir una mayor identificación temprana de los tumores en riesgo de crecimiento durante el período de observación. Además, en este estudio la edad al momento del diagnóstico y la categoría de riesgo del tumor también fueron factores de riesgo independientes para el crecimiento del tumor. Estos hallazgos apoyaron los resultados obtenidos en las poblaciones japonesas y coreanas con respecto a la seguridad de la VA, aunque es importante señalar que la cohorte publicada por el grupo de MSKCC tuvo una media de seguimiento significativamente menor (25 meses). Hasta el año 2019 más de 500 pacientes se han sometido a VA para CPT de hasta $2 \mathrm{~cm}$ de tamaño en el MSKCC, con una mediana de seguimiento de aproximadamente 4 años, y tasas de crecimiento y metástasis ganglionares que siguen siendo comparables a las notificadas en Japón ${ }^{51}$.

En Toronto, Canadá, se realizó un estudio prospectivo observacional con métodos de análisis mixtos (cuantitativos y cualitativos), sobre la toma de decisiones con respecto a la elección de un manejo quirúrgico temprano o VA para el manejo de CPT localizados de bajo riesgo menores a $2 \mathrm{~cm}$ de diámetro máximo ${ }^{52}$. En este estudio, 71\% de los pacientes prefirie- ron VA por sobre cirugía, entre los cuales $94 \%$ eligió de forma independiente qué manejo querían y el resto lo hizo en conjunto con su médico. Los pacientes reportaron que los factores que influyeron en su decisión incluyen la percepción de riesgo de tiroidectomía o de cáncer, consideraciones familiares, momento del tratamiento en el contexto de sus circunstancias de la vida y la confianza en los proveedores de atención de la salud, y el 98\% de los pacientes informó una alta satisfacción de su decisión ${ }^{52}$.

En América Latina, en Colombia, se evaluó el crecimiento del tumor en base al diámetro máximo y se demostró que la tasa de estabilidad general sin crecimiento del tumor mayor a $3 \mathrm{~mm}$ en 12 meses era del 98\% ${ }^{53}$. En Argentina se reportó que a pesar de que sólo el 17\% de los pacientes presentaban un crecimiento tumoral después de un período de seguimiento promedio de 4,6 años, sólo el 25\% aceptaba la VA, y alrededor del 10\% de quienes decidían esta alternativa la interrumpían debido a ansiedad durante el manejo ${ }^{54}$. Más recientemente en Italia, un estudio de una sola institución demostró que sólo tres de 93 pacientes (3,2\%) desarrollaron progresión clínica durante la VA con un seguimiento promedio de 19 meses (rango de 6-54 meses) ${ }^{55}$.

\section{Conclusión}

Los conocimientos adquiridos en los estudios realizados inicialmente en Japón hace tres décadas y más tarde en diversos países con situaciones socioeconómicas y culturales distintas, han demostrado sistemáticamente la seguridad y superioridad de la VA sobre la cirugía temprana o inmediata en pacientes con carcinomas papilares de tiroides de bajo riesgo.

Mediante las extensas experiencias con gran número de pacientes y seguimientos prolongados, se ha evidenciado que la gran mayoría de estos tumores muchas veces no presentan una variación significativa de su tamaño, o con tasas de crecimiento muy lentas e incluso algunos presentan disminución de éste. Además, el grupo minoritario de pacientes que experimentan progresión de su enfermedad y son sometidos a una cirugía de rescate no presentan mayor morbimortalidad que una 
cirugía inmediata o temprana.

Los criterios de selección inicial para la idoneidad deben considerar distintos aspectos, incluyendo las características de los tumores y los pacientes, y la disponibilidad de servicios y equipos médicos especializados. Llevar a cabo una VA no es difícil si se dispone de ecografistas experimentados y si los médicos comprenden con precisión las indicaciones y contraindicaciones de VA.

A pesar de los resultados alentadores la aceptación de la VA en la práctica clínica sigue siendo limitada. En los países emergentes, los fenómenos socioeconómicos y culturales tales como el menor acceso a equipos especializados de salud, ultrasonido de cuello de alta calidad, y resistencia al cambio por parte de algunos médicos, podrían significar mayor dificultad para su desarrollo y práctica.

\section{Bibliografía}

1. Lim H, Devesa SS, Sosa JA, et al. Trends in thyroid cancer incidence and mortality in the United States, 1974-2013. JAMA. 2017; 317:1338-1348.

2. Ito $\mathrm{Y}$, Miyauchi A, Kihara M, et al. Overall survival of papillary thyroid carcinoma patients: a singleinstitution long-term follow-up of 5897 patients. World J Surg. 2018; 42:615-622.

3. Ito Y, Miyauchi A. Prognostic factors and therapeutic strategies for differentiated carcinomas of the thyroid. Endocr J. 2009; 56:177-192

4. Vaccarella S, Franceschi S, Bray F, Wild CP, Plummer M, Dal Maso L. Worldwide thyroid-cancer epidemic? The increasing impact of overdiagnosis. $N$ Engl J Med. 2016; 375(7):614-617.

5. Wiltshire JJ, Drake TM, Uttley L, Balasubramanian SP. Systematic review of trends in the incidence rates of thyroid cancer. Thyroid. 2016; 26(11):1541-1552.

6. Sosa JA, Hanna JW, Robinson KA, Lanman RB. Increases in thyroid nodule fine-needle aspirations, operations, and diagnoses of thyroid cancer in the United States. Surgery. 2013; 154(6):1420-1427.

7. Rego-Iraeta A, Perez-Mendez LF, Mantinan B, Garcia-Mayor RV. Time trends for thyroid cancer in northwestern Spain: true rise in the incidence of micro and larger forms of papillary thyroid carcinoma. Thyroid. 2009; 19(4):333-340.

8. La Vecchia C, Malvezzi M, Bosetti C, et al. Thyroid cancer mortality and incidence: a global overview. Int J Cancer. 2015; 136(9):2187-2195.

9. Kitahara CM, Sosa JA. The changing incidence of thyroid cancer. Nat Rev Endocrinol. 2016; 12(11):646-
653.

10. Albores-Saavedra J, Henson DE, Glazer E, Schwartz AM. Changing patterns in the incidence and survival of thyroid cancer with folicular phenotype-papillary, follicular, and anaplastic: a morphological and epidemiological study. Endocr Pathol. 2007; 18(1):1-7.

11. Davies L, Welch HG. Current thyroid cancer trends in the United States. JAMA Otolaryngol Head Neck Surg. 2014; 140(4):317-322.

12. Burgess JR, Tucker P. Incidence trends for papillary thyroid carcinoma and their correlation with thyroid surgery and thyroid fine-needle aspirate cytology. Thyroid. 2006; 16(1):47-53.

13. Davies L, Welch HG. Increasing incidence of thyroid cancer in the United States, 1973-2002. JAMA. 2006; 295:2164-2167.

14. Ahn HS, Kim HJ, Welch HG. Korea's thyroid-cancer "epidemic"-screening and overdiagnosis. N Engl J Med. 2014; 371:1765-1767.

15. Harach HR, Franssila KO, Wasenius VM. Occult papillary carcinoma of the thyroid. A "normal" finding in Finland. A systematic autopsy study. Cancer. 1985; 56(3): 531-538.

16. Lang W, Borrusch H, Bauer L. Occult carcinomas of the thyroid. Evaluation of 1,020 sequential autopsies. Am J Clin Pathol. 1988; 90(1):72-76.

17. Pelizzo MR, Piotto A, Rubello D, Casara D, Fassina A, Busnardo B. High prevalence of occult papillary thyroid carcinoma in a surgical series for benign thyroid disease. Tumori. 1990; 76(3):255257.

18. Takebe K, Date M, Yamamoto N. Mass screening for thyroid cancer with ultrasonography. Karkinos. 1994; 7:309-317.

19. Jeon MJ, Kim WG, Choi YM, et al. Features predictive of distant metastasis in papillary thyroid microcarcinomas. Thyroid. 2016; 26(1): 161-168.

20. Siddiqui S, White MG, Antic T, et al. Clinical and pathologic predictors of lymph node metastasis and recurrence in papillary thyroid microcarcinoma. Thyroid. 2016; 26(6):807-815.

21. Yu XM, Wan Y, Sippel RS, Chen H. Should all papillary thyroid microcarcinomas be aggressively treated? An analysis of 18,445 cases. Ann Surg. 2011; 254(4):653-660.

22. Adam MA, Pura J, Gu L, et al. Extent of surgery for papillary thyroid cancer is not associated with survival: an analysis of 61,775 patients. Ann Surg. 2014; 260(4):601-607.

23. Barney BM, Hitchcock YJ, Sharma P, Shrieve DC, Tward JD. Overall and cause-specific survival for patients undergoing lobectomy, neartotal, or total thyroidectomy for differentiated thyroid cancer. Head Neck. 2011; 33(5):645-649.

24. Haigh PI, Urbach DR, Rotstein LE. Extent of thyroidectomy is not a major determinant of survival in low- or high-risk papillary thyroid cancer. Ann 
Surg Oncol. 2005; 12(1):81-89.

25. Matsuzu K, Sugino K, Masudo K, et al. Thyroid lobectomy for papillary thyroid cancer: long-term follow-up study of 1,088 cases. World J Surg. 2014; 38(1):68-79.

26. Mendelsohn AH, Elashoff DA, Abemayor E, St John MA. Surgery for papillary thyroid carcinoma: is lobectomy enough? Arch Otolaryngol Head Neck Surg. 2010; 136(11):1055-1061.

27. Nixon IJ, Ganly I, Patel SG, et al. Thyroid lobectomy for treatment of well differentiated intrathyroid malignancy. Surgery. 2012; 151(4):571-579.

28. Cooper DS, Doherty GM, Haugen BR, et al. Revised American Thyroid Association management guidelines for patients with thyroid nodules and differentiated thyroid cancer. Thyroid. 2009; 19(11):1167-1214.

29. Haugen BR, Alexander EK, Bible KC, et al. 2015 American Thyroid Association management guidelines for adult patients with thyroid nodules and differentiated thyroid cancer: the American Thyroid Association guidelines task force on thyroid nodules and differentiated thyroid cancer. Thyroid. 2016;26(1):1-133.

30. Ito Y, Miyauchi A, Kihara M, Higashiyama T, Kobayashi K, Miya A. Patient age is significantly related to the progression of papillary microcarcinoma of the thyroid under observation. Thyroid. 2014; 24(1):27-34.

31. Ito Y, Uruno T, Nakano K, et al. An observation trial without surgical treatment in patients with papillary microcarcinoma of the thyroid. Thyroid. 2003; 13(4): 381-7.

32. Ito $\mathrm{Y}$, Miyauchi $\mathrm{A}$, Inoue $\mathrm{H}$, et al. An observational trial for papillary thyroid microcarcinoma in Japanese patients. World J Surg. 2010; 34(1):28-35.

33. Dahabreh IJ, Chung M, Balk EM, et al. Active surveillance in men with localized prostate cancer: a systematic review. Ann Intern Med. 2012; 156(8):58290.

34. Chung KW. Clinical application of active surveillance in papillary thyroid microcarcinoma. Ann Thyroid. 2017; 2(3):7.

35. Lowenstein LM, Basourakos SP, Williams MD, et al. Active surveillance for prostate and thyroid cancers: evolution in clinical paradigms and lessons learned. Nat Rev Clin Oncol. 2019; 16(3):168-84.

36. Chen RC, Rumble RB, Loblaw DA, et al. Active surveillance for the management of localized prostate cancer (Cancer Care Ontario Guideline): American Society of Clinical Oncology clinical practice guideline endorsement. J Clin Oncol. 2016; 34(18):2182-90.

37. Takami H, Ito Y, Okamoto T, Yoshida A. Therapeutic strategy for differentiated thyroid carcinoma in Japan based on a newly established guideline managed by Japanese Society of Thyroid Surgeons and Japanese
Association of Endocrine Surgeons. World J Surg. 2011; 35:111-21.

38. Japanese Society of Thyroid Surgery, Japan Association of Endocrine Surgeons. Guidelines for the Management of Thyroid Tumors. 2nd ed. Tokyo Japan 2018.

39. Miyauchi A. Clinical trials of active surveillance of papillary microcarcinoma of the thyroid. World J Surg. 2016; 40(3):516-522.

40. Sugitani I, Toda K, Yamada K, et al. Three distinctly different kinds of papillary thyroid microcarcinoma should be recognized: our treatment strategies and outcomes. World J Surg. 2010; 34:1222-1231.

41. Kwon H, Oh HS, Kim M, et al. Active surveillance for patients with papillary thyroid microcarcinoma: a single center's experience in Korea. J Clin Endocrinol Metab. 2017; 102(6):1917-1925.

42. Ito $\mathrm{Y}$, Miyauchi A, Oda H, et al. Revisiting low-risk thyroid papillary microcarcinomas resected without observation: was immediate surgery necessary? World J Surg. 2016;40:523-528.

43. Kakudo K, Tang W, Ito Y, Mori I, Nakamura Y, Miyauchi A. Papillary carcinoma of the thyroid in Japan: subclassification of common type and identification of low risk group. J Clin Pathol. 2004; 57(10):1041-1046.

44. Ito Y, Miyauchi A, Kobayashi K, Miya A. Prognosis and growth activity depend on patient age in clinical and subclinical papillary thyroid carcinoma. Endocr J. 2014; 61(3):205-213.

45. Brito JP, Ito Y, Miyauchi A, Tuttle RM. A clinical framework to facilitate risk stratification when considering an active surveillance alternative to immediate biopsy and surgery in papillary microcarcinoma. Thyroid. 2016; 26(1):144-149.

46. Ito Y, Oda H, Miyauchi A. Insights and clinical questions about the active surveillance of low-risk papillary thyroid microcarcinomas. Endocr J. 2016; 63(4):323-8.

47. Tuttle RM, Fagin JA, Minkowitz G, et al. Natural history and tumor volume kinetics of papillary thyroid cancers during active surveillance. JAMA Otolaryngol Head Neck Surg. 2017; 143(10):1015-20.

48. Sakai T, Sugitani I, Ebina A, et al. Active surveillance for T1b- N0M0 papillary thyroid carcinoma. Thyroid. 2019; 29(1):59-63.

49. Miyauchi A, Kudo T, Ito Y, et al. Estimation of the lifetime probability of disease progression of papillary microcarcinoma of the thyroid during active surveillance. Surgery. 2018; 163(1):48-52.

50. Oh HS, Ha J, Kim HI, et al. Active surveillance of low-risk papillary thyroid microcarcinoma: a multicenter cohort study in Korea. Thyroid. 2018; 28:15871594.

51. Lohia S, Hanson M, Tuttle RM, Morris LGT. Active surveillance for patients with very low-risk thyroid cancer. Laryngoscope Investigative Otolaryngology. 


\section{ARTÍCULO DE REVISIÓN}

2020; 5: 175-182.

52. Sawka AM, Ghai S, Yoannidis T, et al. A Prospective Mixed-Methods Study of Decision Making on Surgery or Active Surveillance for Low-Risk Papillary Thyroid Cancer. Thyroid. 2020;10.1089/ thy.2019.0592.

53. Sanabria A. Active surveillance in thyroid microcarcinoma in a Latin-American cohort. JAMA Otolaryngol Head Neck Surg. 2018; 144:947-948.

54. Smulever A, Pitoia F. Active surveillance in papillary thyroid carcinoma: not easily accepted but possible in Latin America. Arch Endocrinol Metab. 2019; 63: 462-469.

55. Molinaro E, Campopiano MC, Peruzzi L, et al. Active surveillance in papillary thyroid microcarcinoma is feasible and safe: experience at one Italian single center. J Clin Endocrinol Metab. 2019. doi: 10.1210/ clinem/dgz113. 